\title{
Preparation of Cellulose Hydrogel from Sago Pith Waste as a Medium for Seed Germination
}

\author{
Angeline Lecca Wilbert Jampi, ${ }^{1}$ Suk-Fun Chin,${ }^{*}$ Mohd Effendi Wasli ${ }^{1}$ \\ and Chin-Hua Chia ${ }^{2}$ \\ ${ }^{1}$ Faculty of Resource Science and Technology, Universiti Malaysia Sarawak, \\ 94300 Kota Samarahan, Sarawak, Malaysia \\ ${ }^{2}$ School of Applied Physics, Faculty of Science and Technology, \\ Universiti Kebangsaan Malaysia (UKM), 43600 Bangi, Selangor, Malaysia \\ "Corresponding author: sfchin@unimas.my
}

Published online: 25 April 2021

To cite this article: Jampi, A. L. W. et al. (2021). Preparation of cellulose hydrogel from sago pith waste as a medium for seed germination. J. Phys. Sci., 32(1), 13-26. https://doi. org/10.21315/jps2021.32.1.2

To link to this article: https://doi.org/10.21315/jps2021.32.1.2

\begin{abstract}
A large amount of sago pith waste (SPW) produced by the growing sago industry has become an environmental concern. Instead of disposing as waste, SPW could be converted into a hydrogel and used as a seed germination medium. In this study, a hydrogel was prepared from SPW cellulose fibres via crosslinking with epichlorohydrin (ECH). Fourier transform infrared (FTIR) characterisation showed that pure cellulose fibres extracted from the SPW were successfully crosslinked with ECH to form the hydrogel. Scanning electron microscope (SEM) micrograph of the hydrogel showed a porous microstructure. The optimum hydrogel swelling ratio at $19.9 \mathrm{~g} \mathrm{~g}^{-1}$ was obtained with $5 \%$ $w / v$ SPW cellulose content and $5 \mathrm{ml} \mathrm{ECH}$. In this study, the hydrogel hydration property was demonstrated by using it as a medium for maize seed germination. The germination rate (GR) was observed to be above $70 \%$ when SPW cellulose hydrogel alone was used as the medium without the needs for frequent watering.
\end{abstract}

Keywords: Cellulose, hydrogel, sago pith waste, seed germination rate, frequent watering

\section{INTRODUCTION}

Malaysia produces 168 million tonnes of biomass yearly. ${ }^{1}$ Sago industry, for instance, exported approximately 47,000 tonnes of refined sago starch monthly in 2015 alone. $^{2}$ As the sago industry grows, an abundant amount of industrial wastes in the form of effluent and wastewater are being produced and released to 
the nearby areas, leading to environmental pollution. For example, percolation of wastes towards the soil and water streams could occur. Therefore, the utilisation of sago biomass waste is essential for the industry to be sustainable without harming the environment. The component that can be utilised is the sago pith waste (SPW). SPW is defined as the lignocellulosic solid residue of sago palm (Metroxylon sagu). It is the fibrous material left after crushing the sago trunk and removing the starch at the processing. The major SPW contents are starch and lignocellulosic materials mainly consisting of cellulose, hemicelluloses and lignin. ${ }^{3}$ Hence, SPW can potentially be utilised for the production of biofuel and hydrogel. ${ }^{4,5}$ In recent years, attention has been shifted towards utilising biomass wastes for hydrogel production due to their abundant availability, cost-effectiveness and biocompatibility.

A hydrogel is a three dimensional and cross-linked polymer hydrophilic polymeric material that shows gelling properties in the presence of an aqueous medium. ${ }^{6}$ The hydrophilic nature of hydrogel gives rise to its ability to absorb water and contributes to its high swelling ratio. ${ }^{7}$ Meanwhile, its insolubility is due to its cross-linked network chains. ${ }^{8}$ Another property of hydrogel is its capability to swell by absorbing a large amount of water. ${ }^{5,9}$ Even though it swells and absorbs a large amount of water, the hydrogel will preserve its physical structure. This hydrogel property is influenced by its hydrophilicity, crosslink density, ion content, temperature and the ionic strength of the swelling medium.

SPW is suitable as the starting material for the synthesis of the cellulose-based hydrogel. Cellulose is suitable for making hydrogel because it contains many hydroxyl groups. The usage of SPW for the preparation of hydrogel is desirable as it is biomass that is sustainable and renewable. Previous works demonstrate that hydrogel can be prepared from cellulose extracted from biomass such as rice straw, cotton linter pulp, etc. ${ }^{10,11}$

Most hydrogels are being used in agriculture for their water retention properties in soil. ${ }^{12,13}$ Besides, their applications in agriculture are driven by their water storage and controlled release properties. Hydrogels have been used in agriculture as agrochemicals releaser, through which plants can obtain an exact supply of nutrients required. ${ }^{14}$ Excessive nutrients and subsequent wastage and pollution can therefore be avoided. Besides, certain areas that lack water may persist in their agriculture activities through the application of hydrogel.

In this study, a cellulose-based hydrogel was prepared from cellulose fibres isolated from SPW with epichlorohydrin $(\mathrm{ECH})$ used as the crosslinker. SPW cellulose was selected as the precursor material for hydrogel preparation as it is abundantly available, cheap, biocompatible and non-toxic. ${ }^{15}$ Crosslinking of 
cellulose is necessary to enhance the non-solubility, mechanical strength and rigidity of hydrogel. ${ }^{16}$ The potential application of SPW cellulose-based hydrogel potential medium for seed germination was evaluated.

\section{EXPERIMENTAL}

\subsection{Materials and Reagents}

Raw SPW was obtained from CRAUN Research Sdn. Bhd. in Mukah (Sarawak, Malaysia). Urea was procured from J. T. Baker (Philipsburg, United States). Absolute ethanol was supplied by $\mathrm{HmbG}{ }^{\circledR}$ Chemicals (Germany). Sodium hydroxide $(\mathrm{NaOH})$ was purchased from QREC (Asia) (Selangor, Malaysia). Thiourea and acetic acid $\left(\mathrm{CH}_{3} \mathrm{COOH}\right)$ were both supplied by Bendosen. Sodium chlorite $\left(\mathrm{NaClO}_{2}\right)$ was procured from Acros Organics (New Jersey, United States) and ECH from Aldrich (Wisconsin, United States). All chemicals were used without further purification. Maize seeds were bought at a local store in Kota Samarahan (Sarawak, Malaysia). Ultrapure water (UPW) with a resistivity of 18.2 $\mathrm{M} \Omega \mathrm{cm}^{-1}$ was obtained from the Water Purifying System, ELGA, Model Ultra Genetic (United Kingdom) and used throughout the experiments.

\subsection{Extraction of Cellulose Fibres from SPW}

The extraction of cellulose fibres from SPW was done according to the method developed by Gu and Hsieh. ${ }^{17}$ SPW was first ground to fine powders and treated with $\mathrm{NaOH} 4 \% \mathrm{w} / \mathrm{v}$ under stirring at $70^{\circ} \mathrm{C}$ for $1 \mathrm{~h}$ to remove the hemicellulose content. The procedure was repeated twice. Treated samples were then delignified in acidified $\mathrm{NaClO}_{2}$ solution at $70^{\circ} \mathrm{C}$ for $5 \mathrm{~h}$. The $\mathrm{pH}$ of $\mathrm{NaClO}_{2}$ was adjusted to $\mathrm{pH}$ 3-4 with $\mathrm{CH}_{3} \mathrm{COOH}$. The resulting cellulose fibres were washed with deionised water until the $\mathrm{pH}$ is neutral and dried in an oven.

\subsection{Preparation of Hydrogel}

The hydrogel was prepared according to procedures developed by Zhang et al. and Salleh et al. ${ }^{11,18}$ Extracted cellulose fibres were dissolved in an aqueous-based solvent system of $\mathrm{NaOH} /$ urea (NU) of $7 \%$ and $12 \% \mathrm{w} / \mathrm{v}$, respectively. ${ }^{19,20}$ The NU solvent was precooled to $0^{\circ} \mathrm{C}$ before usage. Various weights of cellulose fibres were then dissolved in the NU solvent system and stirred for $1 \mathrm{~h}$. A total of $5 \mathrm{ml}$ of $99 \% \mathrm{ECH}$ was then added dropwise to the mixture to crosslink the cellulose fibres. This amount is used according to the literature, and the optimisation done has found that this amount is effective. Subsequently, the mixture was cooled in a freezer to $-20^{\circ} \mathrm{C}$ for $24 \mathrm{~h}$ until a frozen mass was obtained. The hydrogel was 
obtained upon thawing the frozen mass at room temperature and was washed multiple times with ultrapure water to remove excess chemicals. The hydrogel was then dried in a desiccator and kept for further characterisation.

\subsection{Characterisation of Samples}

Fourier transform infrared (FTIR) spectra of the samples were generated using a FTIR Spectrometer, Thermo Scientific Nicolet iS 10 (United States). The samples were scanned at a resolution of $4 \mathrm{~cm}^{-1}$ within the wavenumber range of 4000 $500 \mathrm{~cm}^{-1}$. The morphology of samples was observed using a scanning electron microscope (SEM), JEOL JSM-6390LA (Japan). The samples were dried at room temperature and then coated with a platinum layer using an Auto Fine Coater (JEOL JFC-1600). Thermogravimetric analysis (TGA) of SPW cellulose hydrogel samples $(2 \mathrm{mg})$ was performed with a thermogravimetric analyser, Perkin-Elmer (United States) under nitrogen gas. For thermal degradation analysis, the samples were heated from ambient temperature to $600^{\circ} \mathrm{C}$ with a heating rate of $10^{\circ} \mathrm{C} \mathrm{min}^{-1}$.

\subsection{Swelling Ratio}

The swelling property of the hydrogel sample was determined according to procedures by Zhang et al. ${ }^{11}$ The pre-weighed dried hydrogel sample was immersed in ultrapure water at room temperature until equilibrium was achieved, where no more changes in weight occurred. ${ }^{11}$ At a time interval of two hours, samples were taken out from the water, dried with delicate wipers, Kimwipe (Canada), and weighed. The swelling ratio was calculated using Equation 1:

$$
\text { Swelling ratio }=\left(W_{S}-W_{d}\right) / W_{d}
$$

where $W_{S}$ and $W_{d}$ are the weight of swollen and dried hydrogel in grams (g), respectively.

\subsection{Seed Germination Rate}

Seed germination study was done according to the method developed by Tao et al. ${ }^{21}$ The maize seeds were washed twice before usage. Then, five Petri dishes were prepared by adding two pieces of wet filter paper. Five different treatments were used, namely A, B, C, D and E, with descriptions for each treatment presented in Table 1. Ten maize seeds were then added to each Petri dish. The Petri dishes were left at room temperature $\left(25^{\circ} \mathrm{C}\right)$ for 7 days with no humidity recorded. Subsequently, $2 \mathrm{ml}$ of water was added every day as a daily feed and was not considered the initial setup. The germination rate of maize was calculated using Equation 2: 


$$
\operatorname{GR}(\%)=\left(G_{t} / T\right) \times 100
$$

where $G_{t}$ is the number of germinated seeds after 7 days, and $T$ is the total number of tested seeds. The test was done in three replicates, and the mean was used in calculations. The data collected was analysed statistically using ANOVA.

Table 1: The experimental design and treatment for maize seeds germinate test.

\begin{tabular}{cccc}
\hline Treatment & Water $(\mathrm{g})$ & Swollen hydrogel $(\mathrm{g})$ & Dry gel $(\mathrm{g})$ \\
\hline $\mathrm{A}$ & 5 & - & - \\
$\mathrm{B}$ & 0 & 5 & - \\
$\mathrm{C}$ & - & 10 & - \\
$\mathrm{D}$ & 5 & - & 0.055 \\
E & 10 & - & 0.085 \\
\hline
\end{tabular}

\section{RESULTS AND DISCUSSION}

\subsection{FTIR Spectra Analysis}

The FTIR spectra of SPW cellulose, the ECH crosslinker and the hydrogel are shown in Figure 1. SPW cellulose hydrogel was obtained through the crosslinking of polymer chains of the cellulose with $\mathrm{ECH}$ catalysed by $\mathrm{NaOH}$. It results from the reaction between $\mathrm{OH}$ groups of cellulose and $\mathrm{ECH}$, together with the self-association and entanglement of cellulose chains via hydrogen bond. From Figure 1(b), it is confirmed that at $3002 \mathrm{~cm}^{-1}$, ECH does contain $\mathrm{OH}$ in its structure. The peak of $1435 \mathrm{~cm}^{-1}$ is attributed to $\mathrm{COO}^{-}$symmetric stretching of the carboxyl group vibration. ${ }^{22}$ This carboxyl group led to hydrogen bonding for the formation of a hydrogel. ${ }^{23}$ The peak observed at $1626 \mathrm{~cm}^{-1}$ for the SPW cellulose had shifted to the lower wavenumber of $1610 \mathrm{~cm}^{-1}$ for the hydrogel, and it was less intense. This peak was attributed to adsorbed water which only existed in the amorphous region of cellulose. ${ }^{24}$ Thus, it could be said that pure cellulose was extracted from SPW after pre-treatment and subsequently interacted with ECH to cause shifting of peak observed in the hydrogel spectrum. The absorption peak at $2886 \mathrm{~cm}^{-1}$ of the SPW cellulose attributed to C-H symmetric stretching, had shifted to the lower wavenumber of $2875 \mathrm{~cm}^{-1}$ in the hydrogel spectrum, indicating successful crosslinking of the hydroxyl group of the SPW cellulose. ${ }^{25}$ Other peaks at $3281 \mathrm{~cm}^{-1}$ and $3362 \mathrm{~cm}^{-1}$ observed for the SPW cellulose and hydrogel spectra were attributed to the $\mathrm{OH}$ stretching. ${ }^{26}$ There were also peaks observed at $1020 \mathrm{~cm}^{-1}$ and $1034 \mathrm{~cm}^{-1}$ of SPW cellulose and hydrogel contributed to C-O 
asymmetric stretching vibration which was common in cellulose, hemicellulose and aryl $\mathrm{OH}$ group of lignin. ${ }^{27}$

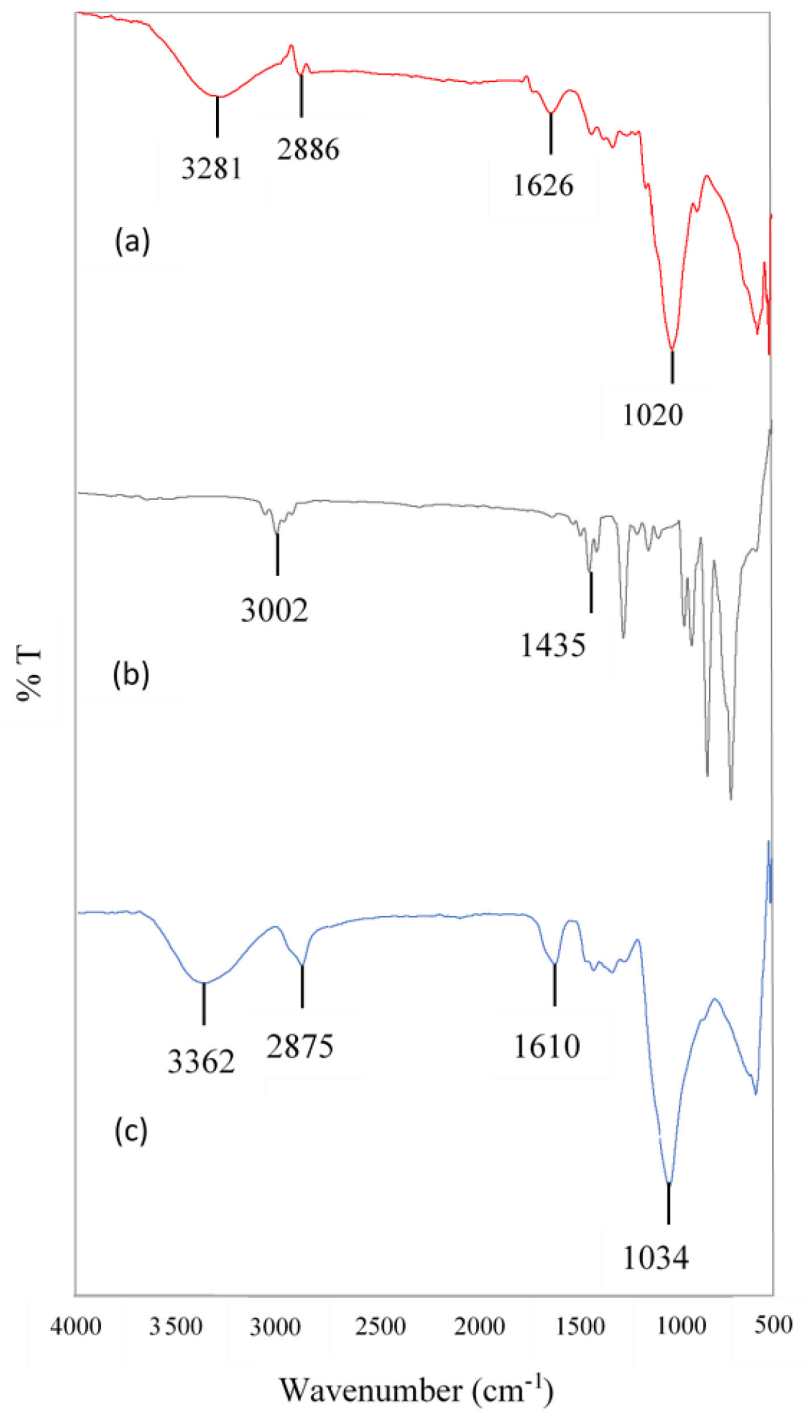

Figure 1: FTIR spectra of (a) SPW cellulose, (b) ECH, and (c) SPW cellulose-ECH hydrogel. 


\subsection{Swelling Kinetic Studies}

The hydrogel and its cellulose fibres were studied visually by using SEM as in Figure 2. The morphology of the extracted cellulose fibre is shown in Figure 2(a) and was found to be $43.8 \mu \mathrm{m}$ in diameter. The successfully synthesised hydrogels showed their microstructure, as presented in Figures 2(b) and (c). The hydrogel was further characterised for their swelling kinetics, with the result presented as a graph showing a 5\% error, shown in Figure 3. From the figure, the highest swelling ratio of $19.7 \mathrm{~g} \mathrm{~g}^{-1}$ was achieved with the optimum condition of $5 \% \mathrm{w} / \mathrm{v}$ SPW cellulose with $5 \mathrm{ml} \mathrm{ECH}$. The highest swelling ratio of $5 \% \mathrm{w} / \mathrm{v}$ SPW hydrogel could be contributed by the presence of pores in its structure, as shown in Figure 2(c). As the hydrogel was frozen and thawed during synthesis, the process has allowed phase separation of the polymer solution, resulting in the porous structure. This could happen when the available water molecules were frozen together and formed openings upon thawing. ${ }^{19}$ The porous structure of the hydrogel was important as it allowed water to enter through its permeation region, leading to the swelling of hydrogel. ${ }^{30,31}$ For $6 \% \mathrm{w} / \mathrm{v}$ SPW cellulose, the swelling ratio was decreased to $8.1 \mathrm{~g} \mathrm{~g}^{-1}$. The decrease in swelling ratio could be explained as the cellulose content was increased, its chains were easily self-entangled with intra and intermolecular hydrogen bonds. Thus, there was less space available for pore swelling. ${ }^{32}$ For samples with the lower percentage of SPW cellulose, $3 \%$ and $4 \% \mathrm{w} / \mathrm{v}$, the swelling ratios were $2.4 \mathrm{~g} \mathrm{~g} \mathrm{~g}^{-1}$ and $2.0 \mathrm{~g} \mathrm{~g}^{-1}$, respectively. For the 3\% w/v SPW cellulose sample, no pore was visible, as shown in Figure 2(b). The SEM images were provided at the highest possible magnification to show the pore's availability. From the images, it is in accord with that as fewer pores were available in hydrogel samples of lower percentage cellulose, such hydrogels were observed to show lower swelling ratios. There are a few factors that can affect the porosity of hydrogel. Techniques like the supercritical drying help retain the pores and make them more available for swelling and viewing. ${ }^{6}$ As the hydrogels were dried in the absence of a supercritical dryer, lower percentage cellulose hydrogel has fewer pores available and less effective to swell. Thus, they exhibited low swelling ratio and non-porous structure in their SEM images. 


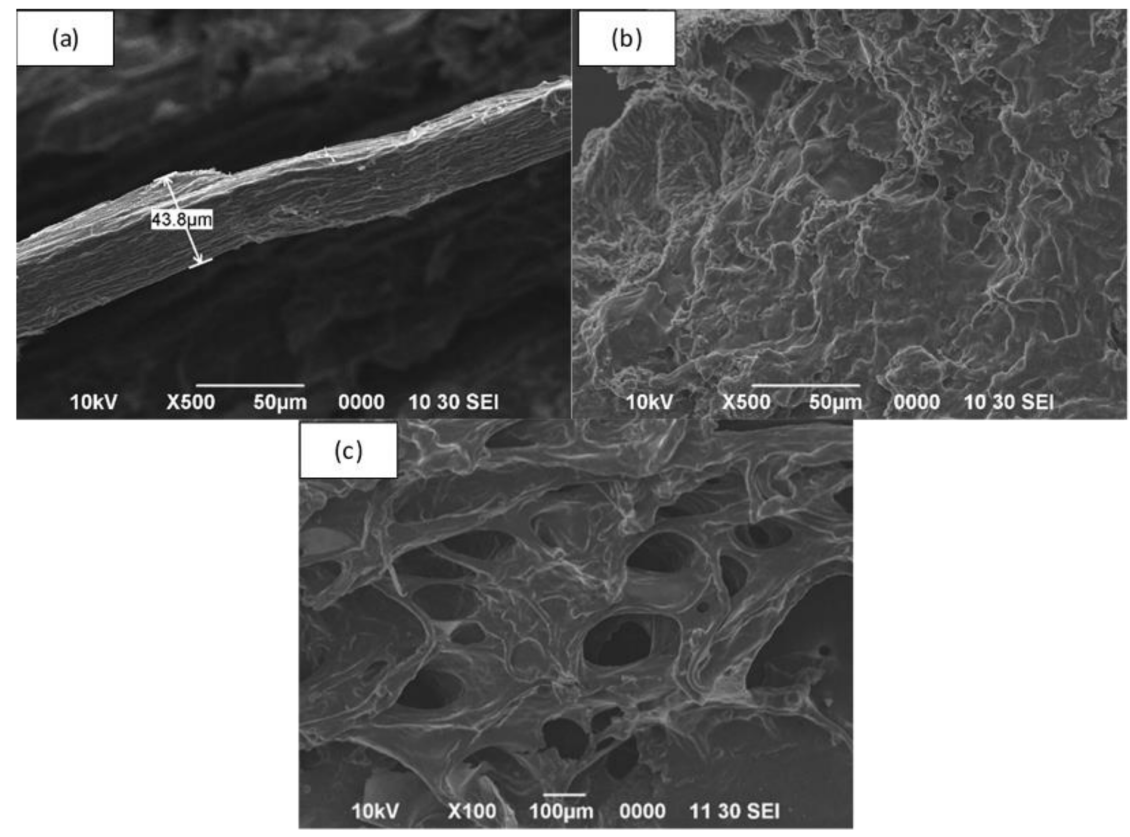

Figure 2: SEM image of (a) SPW cellulose, (b) 3\% w/v SPW cellulose-ECH hydrogel, and (c) $5 \% \mathrm{w} / \mathrm{v}$ SPW cellulose-ECH hydrogel.

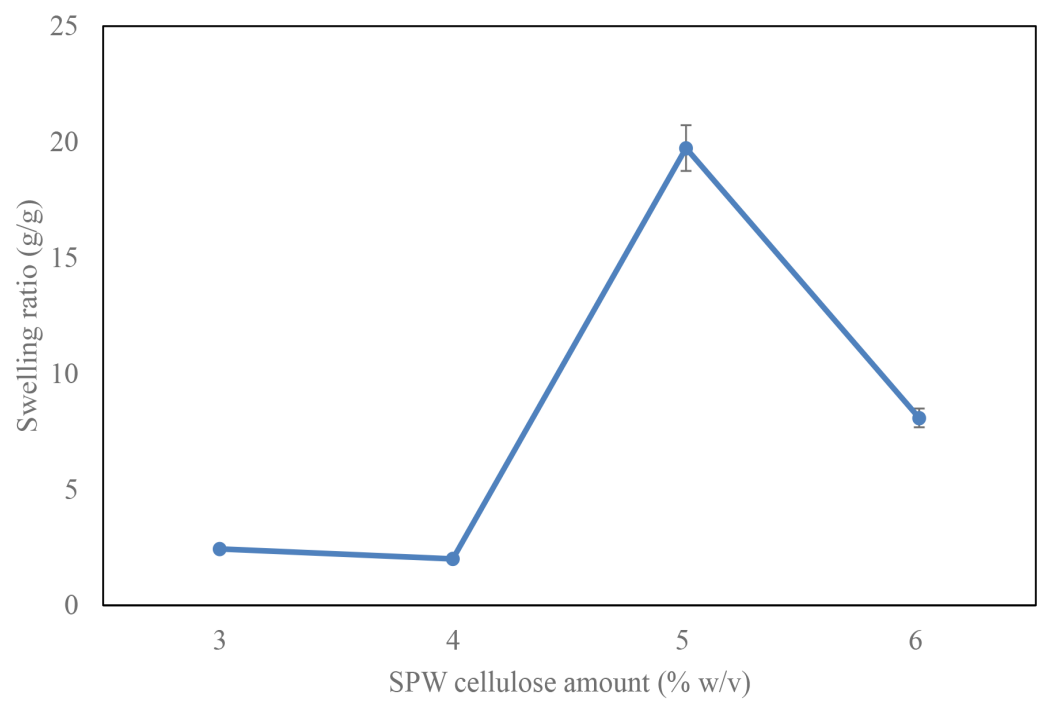

Figure 3: The effect of SPW cellulose amount on the swelling ratio of SPW cellulose-ECH hydrogel. 


\subsection{Thermogravimetric Analysis}

The thermal properties of hydrogel were measured by thermogravimetric analysis. The TG/DTG curves are shown in Figure 4. It was observed that the hydrogel shows a good heat resistance up to around $250^{\circ} \mathrm{C}$. The curves also show that the hydrogel exhibited weight loss and rapidly decreasing weight beyond $250^{\circ} \mathrm{C}$. This rapid pyrolysis occurred due to the dehydration and depolymerisation of the hydrogel. ${ }^{33}$ The DTG curve is the derivative weight percent of TG. It appeared as a peak, thus amplifying small changes in the TG curve. The existence of a peak at $300^{\circ} \mathrm{C}-400^{\circ} \mathrm{C}$ of DTG signifies that there was weight loss occurring at that temperature range. It confirms the TG curve where a rapid and consistent weight loss was observed at the same temperature range, and thus the peak was very obvious and defined.

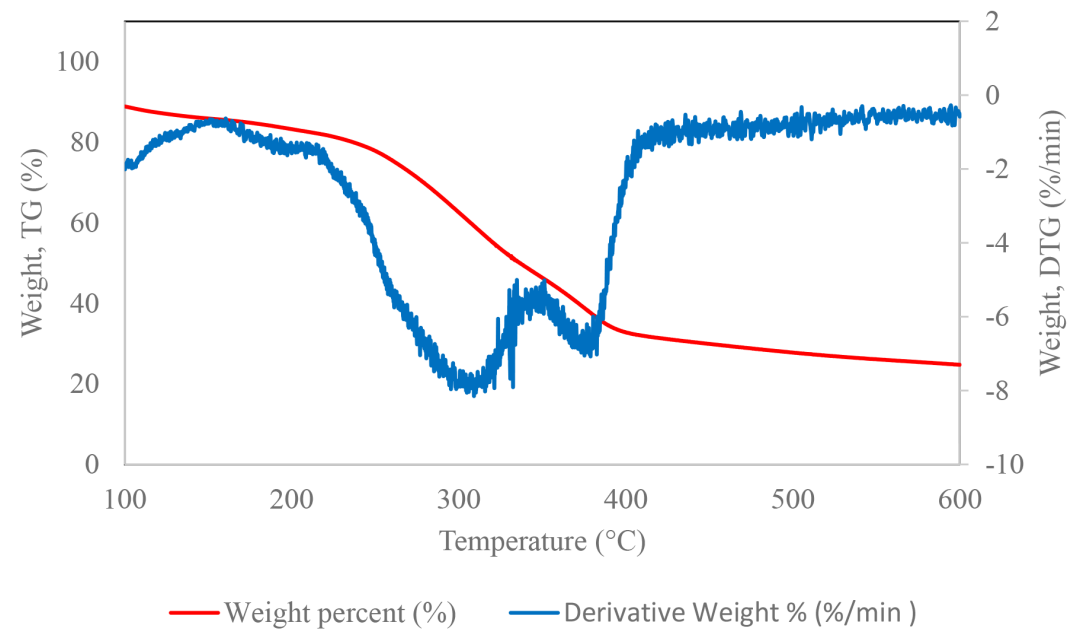

Figure 4: TG curve of $5 \%$ w/v SPW cellulose-ECH hydrogel.

\subsection{Seed Germination Rate}

The effect of different treatments on the seed germination rate of maize is presented in Figure 5 and visually in Figure 6. Based on the results, the maize seeds were germinated in all treatments tested with the germination rate well above $70 \%$ at the end of the experiment, occurring on the seventh day. Based on the ANOVA analysis, no significant difference $(p>0.05)$ in germination rate percentages was observed between the different treatments and the control. On this account, from treatment $\mathrm{D}$, the hydrogel can be used alongside the water in seed germination. As 
for treatments $\mathrm{A}$ and $\mathrm{C}$, applying swollen hydrogel as a medium had a similar effect as adding water directly to the maize seeds for germination. Besides, for treatment $\mathrm{B}$ and $\mathrm{E}$, although the GR percentages were lower as compared with the control (treatment A), no significant difference $(p>0.05)$ in GR percentage between both treatments of medium and the control was observed. In other words, these treatments did not show any significant inhibitory effect on seed germination. Both treatments had allowed the maize seeds to germinate accordingly. These results concurred with previous studies with a hydrogel that demonstrated productive effects on plant growth or promoting seed germinations. ${ }^{12,21}$ Generally, the addition of hydrogel around a plant or seed would increase access to water and provide a longer period of water availability. ${ }^{12}$ In particular, for germinating seeds, hydrogel's presence would increase the water reservoirs near the roots and promote their growth. This is mainly because the seed germination process relies on water, which is known as seed imbibition. During seed imbibition, the dry maize seeds uptake water, subsequently leading to the seeds' radicle penetration. As reported in the literature, several phases of water uptake are involved. The seeds swell and change shape due to initial rapid water uptake, followed by the initiation of embryo expansion. ${ }^{34}$ Finally, the mobilisation of storage reserves are deposited in the storage organs of seeds. The water preserved will allow the mobilisation and consequently leads to seedling growth. Therefore, the presence of hydrogel medium which could swell and shrink accordingly acted as a water reservoir for boosting the maize seed germination. SPW cellulose hydrogel could potentially be used as a cost-effective medium for seed germination in the absence of regular irrigation and promote germination and growth of maize seeds.

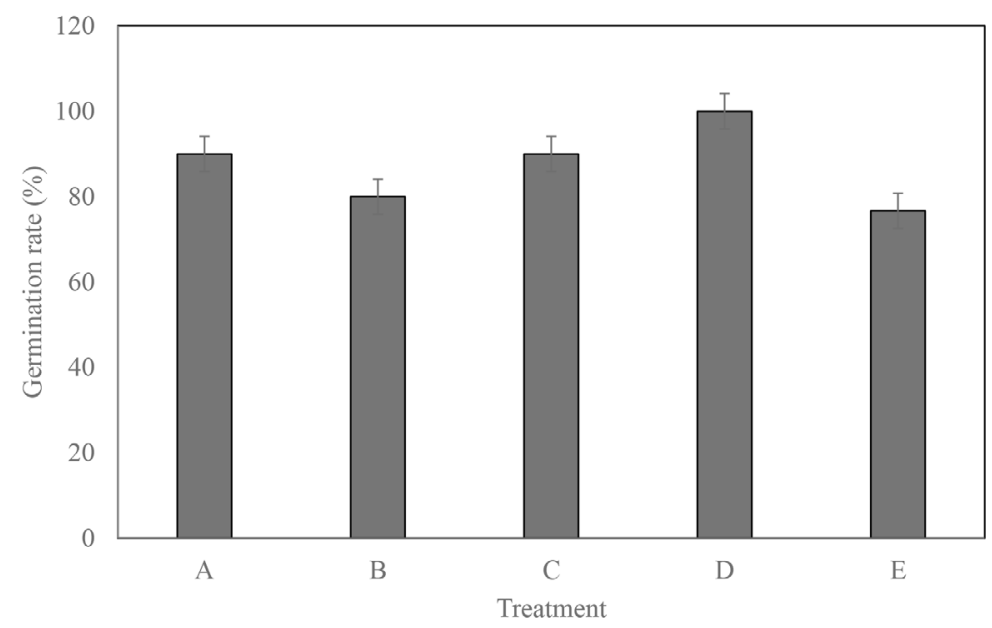

Figure 5: The effect of 5\% w/v SPW cellulose-ECH hydrogel on maize seed germination rate. 

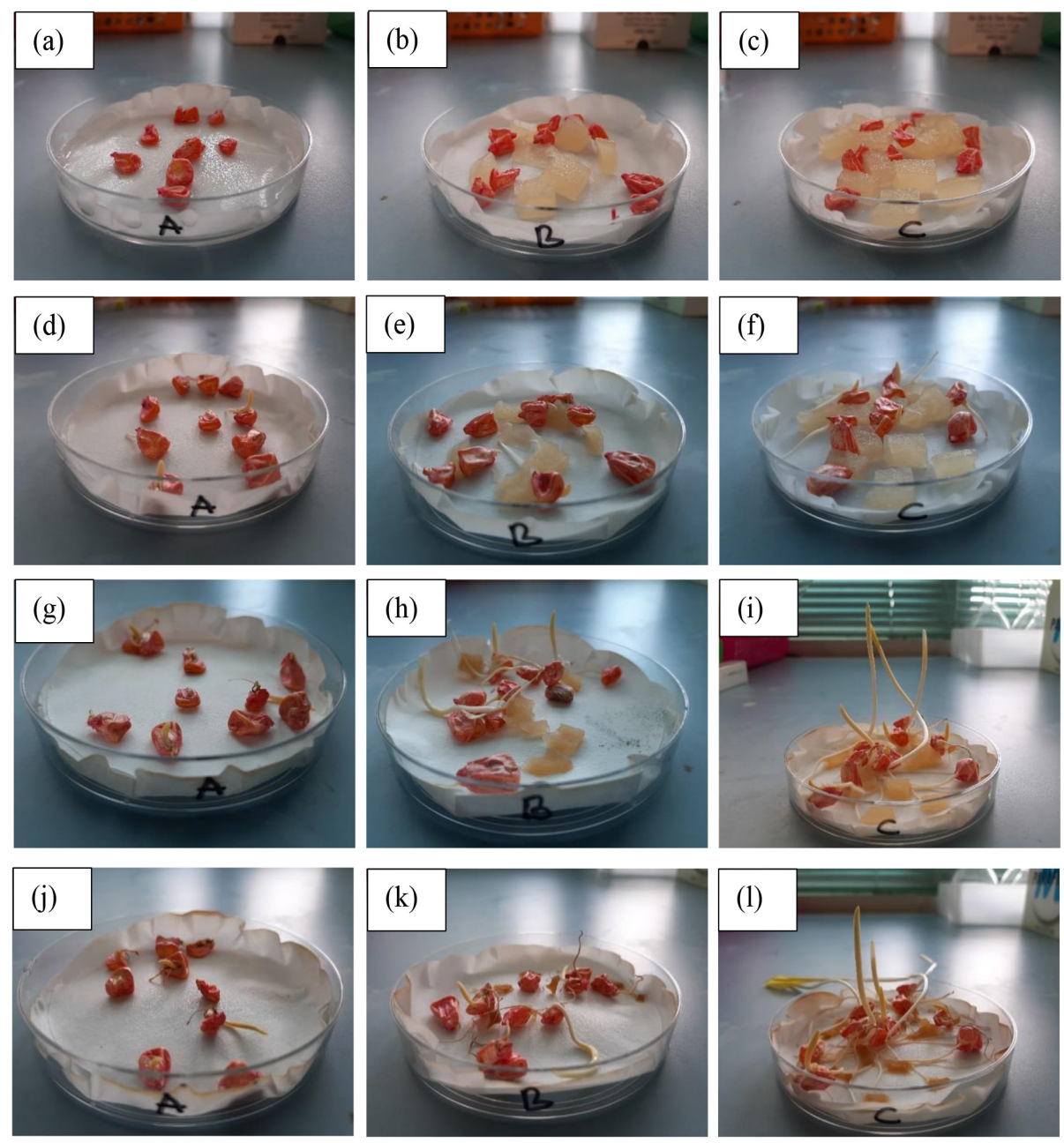

Figure 6: Photographs of maize seed germination test under treatments $\mathrm{A} B$, and $\mathrm{C}$ after one day $(\mathrm{a}-\mathrm{c})$, three days $(\mathrm{d}-\mathrm{f})$, five days $(\mathrm{g}-\mathrm{i})$, and seven days $(\mathrm{j}-\mathrm{l})$.

\section{CONCLUSION}

Cellulose hydrogel was successfully prepared from cellulose fibres extracted from SPW using ECH as a crosslinker. The cellulose molecules cross-linked with each other to form a hydrogel network that exhibited porous network morphology with high swelling ratios. The hydration property of optimised hydrogel was evaluated with the maize seeds showing that the SPW-derived hydrogel possesses high 
potential as a cost-effective and renewable seed germination medium. The usage of such hydrogel as the seed germination medium has shown to reduce the needs for frequent watering during the germination process.

\section{ACKNOWLEDGEMENTS}

The authors gratefully acknowledged the financial support rendered by the Tun Openg Research Grant (F07/TOC/1510/2016) and the International Foundation of Science (grant no. GL/F07/IFS/2018).

\section{REFERENCES}

1. Goh, C. H. (2018). Green energy source going to waste. The Star Online, 24 September. Retrieved from https://www.thestar.com.my/business/ smebiz/2018/09/24/green-energy-source-going-to-waste/

2. Jong, F. S. (2018). An overview of sago industry development, 1980s-2015. In H. Ehara, Y. Toyoda, \& D. V Johnson (eds.), Sago palm: Multiple contributions to food security and sustainable livelihoods. London: Springer, 75-89.

3. Oliveira, J. P. D. et al. (2017). Cellulose fibers extracted from rice and oat husks and their application in hydrogel. Food Chem., 221, 153-160. https://doi.org/10.1016/j. foodchem.2016.10.048

4. Ibrahim, M. F., Kim, S. W. \& Abd-Aziz, S. (2018). Advanced bioprocessing strategies for biobutanol production from biomass. Renew. Sustain. Energy Rev., 91, 1192-1204. https://doi.org/10.1016/j.rser.2018.04.060

5. Li, X. et al. (2016). Characterization, swelling and slow-release properties of a new controlled release fertilizer based on wheat straw cellulose hydrogel. J. Taiwan Inst. Chem. Eng., 60, 564-572. https://doi.org/10.1016/j.jtice.2015.10.027

6. Shen, X. et al. (2015). Hydrogels based on cellulose and chitin: Fabrication, properties, and applications. Green Chem., 18(1), 53-75. https://doi.org/10.1039/ c5gc02396c

7. Zhang, H. et al. (2017). A facile and efficient strategy for the fabrication of porous linseed gum/cellulose superabsorbent hydrogels for water conservation. Carbohydr. Polym., 157, 1830-1836. https://doi.org/10.1016/j.carbpol.2016.11.070

8. Ahmed, E. M. (2015). Hydrogel: Preparation, characterization, and applications: A review. J. Adv. Res., 6(2), 105-121. https://doi.org/10.1016/j.jare.2013.07.006

9. Xu, Q. et al. (2016). A facile route to prepare cellulose-based films. Carbohydr. Polym., 149, 274-281. https://doi.org/10.1016/j.carbpol.2016.04.114

10. Ibrahim, M. M., Abd-Eladl, M. \& Abou-Baker, N. H. (2015). Lignocellulosic biomass for the preparation of cellulose-based hydrogel and its use for optimizing water resources in agriculture. J. Appl. Polym. Sci., 132(42). https://doi.org/10.1002/ app. 42652 
11. Zhang, H. et al. (2017). Cellulose anionic hydrogels based on cellulose nanofibers as natural stimulants for seed germination and seedling growth. J. Agric. Food Chem., 65(19), 3785-3791. https://doi.org/10.1021/acs.jafc.6b05815

12. Montesano, F. F. et al. (2015). Biodegradable superabsorbent hydrogel increases water retention properties of growing media and plant growth. Agric. Agric. Sci. Proced., 4, 451-458. https://doi.org/10.1016/j.aaspro.2015.03.052

13. Mohammadi-Khoo, S. et al. (2016). Synthesis of a cellulose-based hydrogel network: Characterization and study of urea fertilizer slow release. J. Appl. Polym. Sci., 133(5), 1-9. https://doi.org/10.1002/app.42935

14. Gonçalves, A. A. L. et al. (2016). Synthesis and characterization of high performance superabsorbent hydrogels using bis[2-(methacryloyloxy)ethyl] phosphate as crosslinker. Expr. Polym. Lett., 10(3), 248-258. https://doi. org/10.3144/expresspolymlett.2016.23

15. Miyamoto, H., Schnupf, U. \& Brady, J. W. (2014). Water structuring over the hydrophobic surface of cellulose. J. Agric. Food Chem., 62(46), 11017-11023. https://doi.org/10.1021/jf501763r

16. Akhtar, M. F., Hanif, M. \& Ranjha, N. M. (2016). Methods of synthesis of hydrogels: A review. Saudi Pharm. J., 24(5), 554-559. https://doi.org/10.1016/j. jsps.2015.03.022

17. Gu, J. \& Hsieh, Y. L. (2017). Alkaline cellulose nanofibrils from streamlined alkali treated rice straw. ACS Sustain. Chem. Eng., 5(2), 1730-1737. https://doi. org/10.1021/acssuschemeng.6b02495

18. Salleh, K. M. et al. (2018). Chemically crosslinked hydrogel and its driving force towards superabsorbent behaviour. Int. J. Biol. Macromol., 118, 1422-1430. https://doi.org/10.1016/j.ijbiomac.2018.06.159

19. Voon, L. K., Cem, S. Pang, S. C. \& Chin, S. F. (2016). Highly porous cellulose beads of controllable sizes derived from regenerated cellulose of printed paper wastes. Mater. Lett., 164, 264-266. https://doi.org/10.1016/j.matlet.2015.10.161

20. Voon, L. K., Pang, S. C. \& Chin, S. F. (2016). Regeneration of cello-oligomers via selective depolymerization of cellulose fibers derived from printed paper wastes. Carbohydr. Polym., 142, 31-37. https://doi.org/10.1016/j.carbpol.2016.01.027

21. Tao, J. et al. (2018). Effects of eco-friendly carbohydrate-based superabsorbent polymers on seed germination and seedling growth of maize. Royal Soc. Open Sci., 5(2). https://doi.org/10.1098/rsos.171184

22. Yang, S. et al. (2011). Preparation and release properties of a pH-tunable carboxymethyl cellulose hydrogel/methylene blue host/guest model. Int. J. Polym. Mater. Polym. Biomater., 60(1), 62-74. https://doi.org/10.1080/00914037.2010.5 04160

23. Lin, Q. et al. (2017). Synthesis of magnetic epichlorohydrin cross-linked carboxymethyl cellulose microspheres and their adsorption behavior for methylene blue. J. Environ. Sci. Health A, 52(2), 106-116. https://doi.org/10.1080/1093452 9.2016.1237117

24. Navarra, M. A. et al. (2015). Synthesis and characterization of cellulose-based hydrogels to be used as gel electrolytes. Membr., 5(4), 810-823. https://doi. org/10.3390/membranes5040810 
25. Lin, Q. et al. (2017). Highly effective adsorption performance of carboxymethyl cellulose microspheres crosslinked with epichlorohydrin. J. Appl. Polym. Sci, 134(2), 1-11. https://doi.org/10.1002/app.44363

26. Raucci, M. G. et al. (2014). Effect of citric acid crosslinking cellulose-based hydrogels on osteogenic differentiation. Mater. Res. A, 103A, 2045-2056. https:// doi.org/10.1002/jbm.a.35343

27. Gogoi, G. \& Hazarika, S. (2017). Coupling of ionic liquid treatment and membrane filtration for recovery of lignin from lignocellulosic biomass. Sep. Purif. Technol., 173, 113-120. https://doi.org/10.1016/j.seppur.2016.09.019

28. Xu, Z. et al. (2016). Morphological and swelling behavior of cellulose nanofiber (CNF)/poly(vinyl alcohol) (PVA) hydrogels: Poly(ethylene glycol) (PEG) as porogen. RSC Adv., 6(49), 43626-43633. https://doi.org/10.1039/C6RA03620A

29. Chin, S. F. et al. (2019). Antimicrobial starch-citrate hydrogel for potential applications as drug delivery carriers. J. Drug Del. Sci. Technol., 101239. https:// doi.org/10.1016/j.jddst.2019.101239

30. Pang, S. C. et al. (2011). Starch-maleate-polyvinyl alcohol hydrogels with controllable swelling behaviors. Carbohyd. Polym., 84(1), 424-429, https://doi. org/10.1016/j.carbpol.2010.12.002

31. Tang, H. et al. (2014). Swelling behaviors of superabsorbent chitin/ carboxymethylcellulose hydrogels. J. Mater. Sci., 49(5), 2235-2242. https://doi. org/10.1007/s10853-013-7918-0

32. Chin, S. F., Jimmy, F. B. \& Pang, S. C. (2016). Fabrication of cellulose aerogel from sugarcane bagasse as drug delivery carriers. J. Phys. Sci., 27(3), 159-168. https://doi.org/10.21315/jps2016.27.3.10

33. Phinichka, N. \& Kaenthong, S. (2018). Regenerated cellulose from high alpha cellulose pulp of steam-exploded sugarcane bagasse. J. Mater. Res. Technol., 7(1), 55-65. https://doi.org/10.1016/j.jmrt.2017.04.003

34. Tuan, P. A. et al. (2019). Molecular mechanisms of seed germination. In Feng, H. (eds.), Sprouted grains. New York: AACC, 1-24. https://doi.org/10.1016/b978-012-811525-1.00001-4 